\title{
Assessing the risk of osteonecrosis of the jaw due to bisphosphonate therapy in the secondary prevention of osteoporotic fractures
}

\author{
F. Lapi • F. Cipriani • A. P. Caputi • G. Corrao • \\ A. Vaccheri • M. C. Sturkenboom • M. Di Bari • \\ D. Gregori • F. Carle • T. Staniscia • A. Vestri • \\ M. Brandi • V. Fusco • G. Campisi • G. Mazzaglia • \\ on behalf of the Bisphosphonates Efficacy-Safety Trade- \\ off (BEST) study group
}

Received: 9 February 2012 / Accepted: 14 March 2012

(C) International Osteoporosis Foundation and National Osteoporosis Foundation 2012

\begin{abstract}
Summary There is evidence that the use oral bisphosphonates can lead to osteronecrosis of the jaws (ONJ). Although
\end{abstract}

F. Lapi $\cdot$ F. Cipriani $\cdot$ G. Mazzaglia

Regional Agency for Healthcare Services of Tuscany,

Florence, Italy

F. Lapi

Department of Preclinical and Clinical Pharmacology,

University of Florence,

Florence, Italy

F. Lapi

Centre for Clinical Epidemiology,

Jewish General Hospital,

McGill University,

Montreal, QC, Canada

A. P. Caputi

Department of Medicine and Pharmacology,

University of Messina,

Messina, Italy

G. Corrao

Unit of Biostatistics and Epidemiology, Department of Statistics,

University of Milan-Bicocca,

Milan, Italy

A. Vaccheri

Department of Pharmacology, University of Bologna,

Bologna, Italy

M. C. Sturkenboom · G. Mazzaglia

Department of Medical Informatics, Pharmacoepidemiology Unit, Erasmus University Medical Center,

Rotterdam, The Netherlands the occurrence of ONJ appears rare among oral bisphosphonates (BPs) users, it is important to know that it exists and can be opportunely minimized.

\section{Di Bari}

Department of Critical Care Medicine and Surgery,

Unit of Gerontology and Geriatrics, University of Florence,

Florence, Italy

D. Gregori

Department of Public Health and Environmental Medicine,

University of Padua,

Padua, Italy

F. Carle

Center of Epidemiology, Biostatistics, and Medical Information

Technology, Polytechnic University of Marche,

Ancona, Italy

T. Staniscia

Department of Medicine and Aging, University "G. d'Annunzio",

Chieti-Pescara, Italy

A. Vestri

Department of Public Health and Infectious Disease,

University "La Sapienza",

Rome, Italy

M. Brandi

Department of Internal Medicine, University of Florence,

Florence, Italy

V. Fusco

Oncology Unit, Alessandria Hospital,

Alessandria, Italy 
Introduction The purpose of this study is to evaluate the association between BPs prescribed for the secondary prevention of osteoporotic fractures and the occurrence of ONJ. Methods An Italian record linkage claims database with a target population of around 18 million individuals (6 million over 55 years of age) constituted the data source. We conducted a nested case-control study within a cohort of individuals aged $55+$ years old, who were discharged from hospitals with a primary diagnosis of incident osteoporotic fracture. The date related to the discharge diagnosis of ONJ was the index date. Conditional logistic regression for matched data was fitted to estimate the odds ratio (OR) along with $95 \%$ confidence intervals $(95 \% \mathrm{CI})$ for the likely association between use of BPs and the risk of ONJ. Results Any one of the 61 ascertained cases of ONJ (incidence rate, 36.6 per 100,000 person-years) was matched to 20 controls for a total of 1120 controls. When the exposure to BPs was modeled according to recency (i.e., exposure time window prior to the index date) of use, the adjusted OR (95\% CI) for current users was 2.8 (1.3-5.9) against never users. The cumulative use of BPs has shown to increase the incidence of ONJ among patients with primary osteoporotic fractures, although not statistically significant risk has been observed.

Conclusions Although the risk of BP-related ONJ appears low in non-oncological indications, it is important to be aware that it exists and to know how it may be predicted and possibly minimized.

Keywords Bisphosphonates · Nested case-control study · Osteonecrosis of the jaw · Osteoporotic fractures

\section{Introduction}

Osteonecrosis of the jaw (ONJ), a rare condition characterized by exposed necrotic bone in the maxillofacial region [1, 2], has received increasing attention since case reports among patients exposed to bisphosphonates (BPs) were published in 2003 [3, 4].

The ONJ clinical picture mainly consists of non-healing ulcerated oral lesions and visible necrotic bone, which are

\section{G. Campisi}

Department of Surgical and Oncological Disciplines, Sector of Oral Medicine, University of Palermo,

Palermo, Italy

F. Lapi $(\bowtie)$

Department of Preclinical and Clinical Pharmacology,

University of Florence (Italy),

Viale G. Pieraccini 6,

50139 Florence, Italy

e-mail: francesco.lapi@unifi.it sometimes associated with a diffuse jaw or facial pain [2, 5-7]. The exact etiologic mechanisms remain unclear, but it might relate, in part, to altered bone remodeling or local tissue effects in susceptible patients [1, 8, 9].

Most affected patients have incurred ONJ after invasive dental procedures that involved dental bone manipulation, although spontaneous exposure of bone has been also observed. Cofactors have not been firmly established, although smoking, steroid use, anemia, hypoxemia, diabetes, infections, and immune deficiency may be important [2, 5-7]. Indeed, as only a minority of BPs users develop ONJ, it is conceivable that individual pharmacokinetic and pharmacodynamic genetic variations may confer susceptibility or resistance in developing this complication. Among genetic determinants, polymorphisms on $C Y P 2 C 8$ gene [10] and of $F D P_{c} S$ gene [11] arise as promising risk factors.

To date, most of the reported cases have been related to zoledronic or pamidronic acid when they were administered intravenously to control metastatic bone disease or multiple myeloma [4, 12, 13], for which reported ONJ incidence ranges from 4 to $10 \%$, and the mean time of onset varies from 1 to 3 years $[1,14,15]$.

Early case series also identified approximately $5 \%$ of the reported cases occurring in patients receiving oral BPs $[1,8]$, primarily used for the prevention of osteoporotic fractures. However, limited data exist about the ONJ risk with such medications, and most of the published evidence are derived from surveillance data [16]. A study from Germany, which used information from the Central Registry of ONJ, reported a prevalence among patients treated with BPs for osteoporosis of $0.00038 \%$ [17]. In a survey of 8572 Kaiser Permanente respondents, the prevalence of ONJ in patients receiving long-term (more than 2 years) oral BP therapy ranged from 0.05 to $0.21 \%$, depending on the duration of exposure [18]. Finally, in a nationwide survey of oral and maxillofacial surgeons in Australia, the incidence of ONJ among osteoporotic patient treated weekly with alendronate was $0.01-0.04 \%$. Following dental extractions, this rate increased to $0.09-0.34 \%$ [14]. In addition, inferences from observational epidemiologic studies published so far offer conflicting results, and in most of the cases, they are subjected to unmeasured confounding and biases [19-21].

Given the increasing number of persons taking oral BPs for the treatment of osteopenia/osteoporosis [22], it is important to accurately determine the incidence of ONJ using rigorous case ascertainment criteria and to assess the risk associated with long-term use of such medications. We therefore conducted a nested case-control study to evaluate the possible association between the use of oral BPs prescribed for the secondary prevention of osteoporotic fractures and the occurrence of ONJ. 


\section{Methods}

Data source

The data used for this study were obtained from the Bisphosphonates Effectiveness Safety Trade-off (BEST) network. It collects record linkage encrypted information on patient demographics, hospital discharge schemes (HDS), the related surgical and/or diagnostic procedures [since January 1, 1998 to December 31, 2007; coded by International Classification Disease, 9th version, Clinical Modification (ICD9CM)], any reimbursed drug prescription (since January 1, 2003 to December 31, 2007; coded by Anatomical Therapeutic Chemical classification), and the mortality registers (since January 1, 2003 to December 31, 2007) from a target population of around 18 million people residing in four Italian Regions and in the catchments areas of ten Local Health Authorities (LHA). The study was approved by the Local Research Ethics Committees of each Region or LHA being involved into the project.

\section{Cohort selection}

The process of cohort selection is depicted in Fig. 1. All patients aged 55+years, who were discharged because of an osteoporotic fracture during the period July 1, 2003-December 31, 2005, were identified. Fractures were selected among ICD9CM codes (primary and secondary positions) consistently associated to osteoporotic fractures site, (2327) such as vertebrae (codes: $733.13,805^{*}$ ), pelvis (code: 808*), femur/hip (codes: 733.14, 820*, 821*), and tibia/ fibula (codes: 733.16, 823*) [23-26]. The date of the first recorded HDS of osteoporotic fracture was considered as the "entry date" of follow-up.

Patients were excluded if they had, before the entry date, less than 6 months of tracking period, an evidence of hospitalization for osteoporotic fracture or fractures at multiple sites (i.e., more likely to be associated with non-osteoporotic fracture), Paget's disease (code: $731^{*}$ ), and malignant neoplasms (codes: 140 to 208). The prescription of BPs in the 6 months prior to entry date has been also considered an exclusion criterion. No cases of ONJ (see "Cases and controls definition" section) were captured before the entry date.

Each participant accrued person-years of follow-up from the entry date until the earliest of the following events: ONJrelated diagnosis, occurrence of one of the exclusion criteria (with the exception of osteoporotic fractures), death, transferring out of BEST catchment areas, or end of the study period (December 31, 2007).

Cases and controls definition

Cases were members of the study cohort who had experienced an ONJ event during the follow-up. Since there were no specific ICD9CM codes for the ONJ-related events during the study period, a four-step process identified ONJ cases from the study population.

First, potential ONJ cases have been retrieved for main or secondary diagnosis from ICD9CM and Current Procedural Terminology billing codes from the regional or LHA registries of the HDS. In a preliminary evaluation, a highly sensitive case-finding procedure has been implemented. Subsequently, an adjudication committee composed by a panel of experts in dentistry, oncology, geriatric, and pharmacology selected from the preliminary list only diagnostic codes and procedures that could have been most likely used to describe or treat a necrotic lesion of the jaw. Second, among the selected cases, additional information was collected from the medical charts using a structured questionnaire. The questionnaire included information on descriptive discharge diagnosis, tomography or panoramic imaging, history of dental extraction, tissue biopsy, signs or symptoms. Third, the collected questionnaires were reviewed by the study staff for excluding potential false positives (i.e., patients with direct or indirect evidence of cancer, trauma, osteonecrosis at other bone sites as well as signs and/or symptoms related to the main other oral-maxillary diseases). Fourth, remaining cases were classified by two ONJ experts as "definite" (i.e., 22 cases) if: (1) sign or symptoms suggesting necrotic bone lesions, inflammatory jaw condition not related to strict dental causes, and open lesions/ulcers of the jaw were recorded. All lesions related to soft tissues (e.g., recurrent aphthous ulcers) were purposely identified and excluded; [5] (2) the ONJ was specifically mentioned in the medical chart; and (3) pain was not directly referred to dental extraction or other surgical procedures. The other cases not fulfilling these criteria were classified as "probable" (i.e., 39 cases). For each case, up to 20 controls were randomly selected among the patients' cohort. Controls were matched to their respective case by sex, age ( \pm 3 years), month, and year of the cohort entry. The date of ONJ-related diagnosis was the "index date," and the same date was assigned as the index date to the respective matched controls.

\section{Exposure assessment}

Patients have been considered on BPs treatment once they received a prescription between the entry date and the index date. The number of days supplied from each prescription has been calculated assuming a standard dosing regimen, as assessed by the defined daily dose. It has also been used to calculate the sum of days of BPs exposure (plus 28 days to account for potential hospital prescriptions) during the entire follow-up. For each patient, adherence to BPs was therefore estimated by dividing the sum of days of BPs exposure with the follow-up [medical possession ratio (MPR)], and 


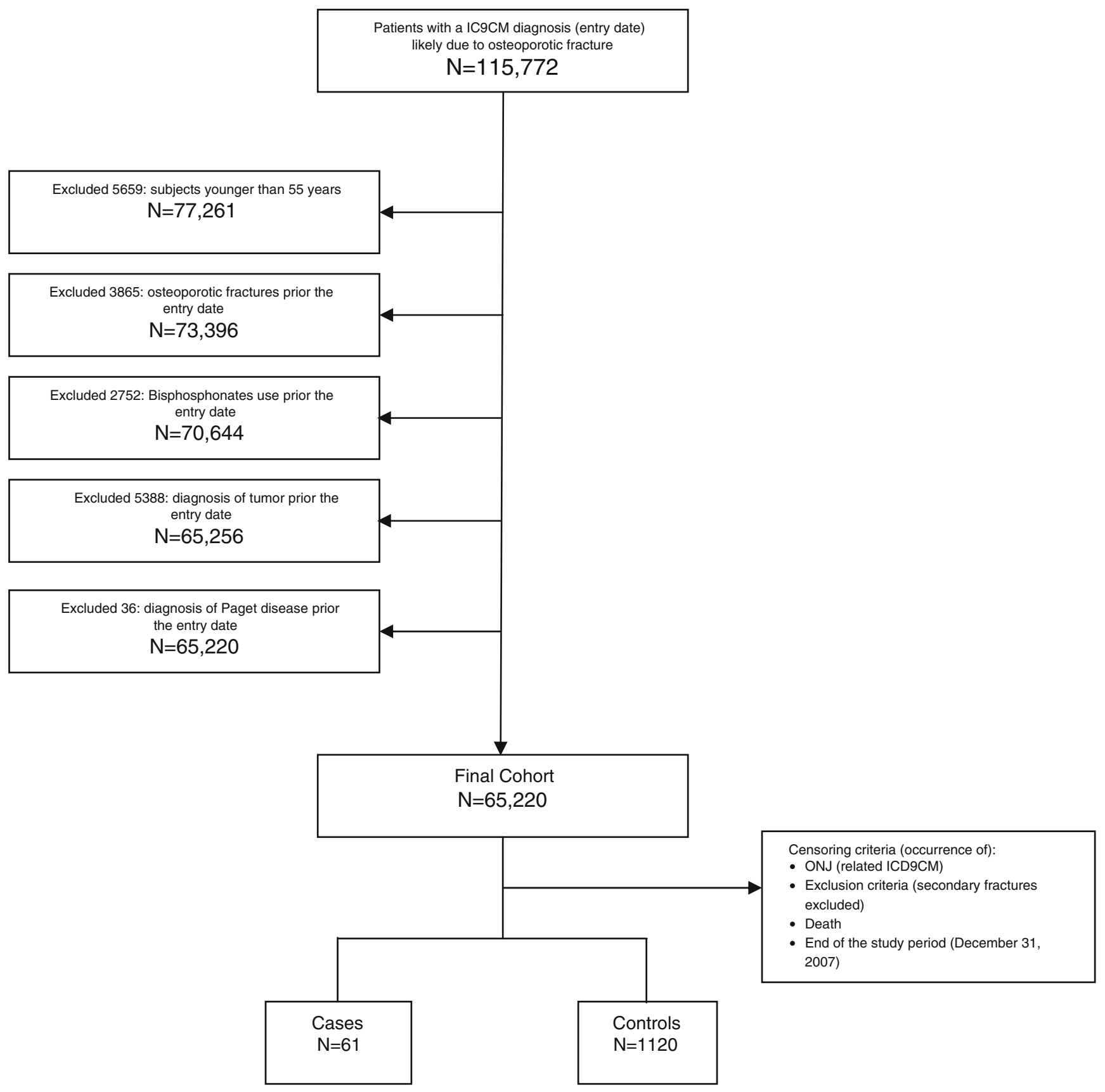

Fig. 1 Flow chart of cohort and case-control selection process

further classified into the following adherence levels: high (MPR $\geq 80 \%$ ), intermediate (MPR $=40-79 \%$ ), and low (MPR $<40 \%$ ).

Following the case and control selection, patients were categorized into mutually exclusive categories of current use, past use, and non-use, according to latest date of BPs pharmacy claims supply before the index date. Current use has been defined as latest date of exposure occurring within 1 year before the index date; past use as exposure interrupted more than 1 year before the index date, and non-use as no recorded BPs prescription at any time during follow-up.
Current users have been further subdivided into four categories (i.e., $\leq 90$ days, from 91 to 179 days, from 180 to 364 days, and $\geq 365$ days) according to the sum of days of BPs exposure during follow-up.

\section{Covariates}

As covariates, we evaluated the patients' demographics (i.e., age and gender) and the presence of risk factors for ONJ as commonly described in the most recent guidelines on ONJ [5, 7]: (1) concurrent drug use [i.e., antithrombotics, proton 
pump inhibitors (PPIs), non steroidal anti-inflammatory drugs (NSAIDs), beta-blockers, antidepressants, antiepileptic drugs, lipid-lowering drugs, calcium and/or vitamin D supplements, immunosuppressant, and corticosteroids; captured within 1 year before the index date]; (2) concurrent diseases (captured any time before the index date), such as diabetes mellitus (code 250* and use of antidiabetic drugs), vascular coagulation disorders (codes 093.0, 286* , 287.1, 287.3 through 287.5, $440^{*}, 441^{*}, 443.1$ through 443.9, 447.1, V43.4), and the Charlson Comorbidity Index (CCI) as index of chronic severity degree. Occurrence of any osteoporotic fracture during follow-up was not censored, and it was considered in the case-control analysis.

\section{Data analysis}

Density method has been used in measuring the incidence of ONJ. The number of events that occurred during follow-up and the person-months cumulatively spent in the corresponding periods by the cohort members constituted the numerator and the denominator of the incidence rates, respectively.

Statistical differences between cases and controls were evaluated by means of $\chi^{2}$ or $t$ tests for categorical and continuous variables, respectively. Conditional logistic regression for matched data was fitted to estimate the odds ratio (OR) along with the corresponding $95 \%$ confidence intervals $(95 \%$ CI), for the association between use of BPs and the risk of ONJ. After Mantel-Haenszel test, the OR estimates were adjusted for those variables significantly (10\%) affecting the crude relationship between BPs and ONJ.

To test the robustness of our findings, some sensitivity analyses were performed. Firstly, the analyses were restricted to definite cases only. Secondly, because the allocation of BPs therapy was not randomized and the indication for treatment may be related to the risk of future health outcomes, a propensity score (PS) matching design was carried out with the aim of taking into account the confounding by indication [27]. The PS was estimated at patient level by performing a multivariable logistic regression. Each known predictor of BPs exposure and ONJ occurrence, as well as those risk factors for ONJ constituted the covariates vector which established the propensity to BPs use. All variables were imputed in the model in a non-parsimonious way according to methodological literature [28]. Afterwards, cases and controls were matched (1:20) by the corresponding PS within \pm 0.1 . Finally, to account for the possible effect dilution due to short-term BPs users, all models were rerun by excluding individuals who begun the therapy less than 6 months before the index date.

All analyses were performed using STATA 10.0 for Windows. Statistical significance was defined as a $p$ value less than 0.05 .

\section{Results}

From the BEST database, 115,772 patients with an HDS for osteoporotic fracture were selected between January 1, 2003 and December 31, 2005. Of these, 65,220 patients aged 55 years and older, after the application of the exclusion criteria, constituted the comprehensive study cohort (Fig. 1).

Incidence rates

During the follow-up, we identified 61 cases of ONJ (22 definite), with an incidence rate of 36.6 (95\% CI, 28.546.9) per 100,000 person-years. An increased incidence has been observed among patients with diabetes mellitus [77.3 (95\% CI, 46.6-128.4)], as well as among those being prescribed corticosteroids during follow-up [58.3 (95\% CI, 24.2-140.1)]. Higher incidence has been also reported among BPs users [46.0 (95\% CI, 24.8-85.5)], when compared with nonusers [35.2 (95\% CIs, 26.8-46.3)]. Among BPs users, we have also observed a growing trend related to the adherence levels, with incidence ranging from 27.3 (95\% CIs, 10.3-72.8) among low adherers (MPR $<40 \%$ ) to 111.7 (95\% CIs, 27.9-446.6) among high adherers $(\mathrm{MPR} \geq 80 \%)$.

\section{Nested case-control study}

All 65,220 eligible patients were followed up (median, 2.7 years), and 61 ONJ cases along with 1120 matched controls were considered for the analysis. The characteristics of the cases and controls at the index date are described in Table 1. Overall, cases were more likely to have comorbid conditions, as assessed by using the CCI, or by diabetes prevalence (27.9 vs. $17 \%$ ). They were also more likely to be prescribed with co-medications such as antithrombotics (50.8 vs. $30.5 \%$ ) and immunosuppressant (4.9 vs. $0.2 \%$ ). After adjustment for selected covariates, we observed a nonstatistically significant increased risk of ONJ (OR, 1.7; 95 \% CI, 0.8-3.6) associated with BPs use. However, when the analysis was restricted to current users, a statistically significant 2.8 -fold increased risk was reported (OR, 2.8; $95 \%$ CI, 1.3-5.9), when compared to nonusers (Table 2). The statistical significance among current users was confirmed in a sensitivity analysis which selected propensity-matched controls (OR, 2.3; $95 \% \mathrm{CI}, 1.1-5.1$ ).

Upon restriction to 22 definite ONJ cases and 440 matched controls, the association maintained similar risk among current users (OR, 2.5; 95 \% CI, 0.7-9.5). In such analysis, also a longer exposure with oral BPs (i.e., 180364 days) showed a clearer trend toward an increased risk of ONJ (OR, 4.2; $95 \%$ CI, 0.5-41.4), although, due to the small sample size, it did not reach the statistical significance. Finally, the exclusion of individuals who started the therapy 
Table 1 Characteristics of case and controls at the index date
$S D$ standard deviation, $C C I$

Charlson Comorbidity Index,

NSAIDs non steroidal antiinflammatory drugs, PPIs proton pump inhibitors

${ }^{a}$ ICD9CM coded diagnosis or an antidiabetic prescription

\begin{tabular}{|c|c|c|c|c|c|}
\hline & \multicolumn{2}{|l|}{ Cases } & \multicolumn{2}{|l|}{ Controls } & \multirow[b]{2}{*}{$p$ value } \\
\hline & $N=61$ & $\%$ & $N=1120$ & $\%$ & \\
\hline Gender, female & 42 & 68.9 & 840 & 68.9 & - \\
\hline Age $($ mean $\pm \mathrm{SD})$ & $73.2 \pm 0.7$ & & $73.1 \pm 9.1$ & & - \\
\hline \multicolumn{6}{|l|}{ Comorbidity } \\
\hline CCI & & & & & 0.001 \\
\hline 0 & 21 & 34.4 & 687 & 56.3 & \\
\hline 1 & 22 & 36.1 & 299 & 24.5 & \\
\hline 2 & 7 & 11.5 & 139 & 11.4 & \\
\hline $3+$ & 11 & 18.0 & 95 & 7.8 & \\
\hline Diabetes mellitus ${ }^{\mathrm{a}}$ & 17 & 27.9 & 207 & 17.0 & 0.029 \\
\hline Osteoporotic fractures (secondary) & 8 & 13.1 & 171 & 14.0 & 0.843 \\
\hline Vascular and coagulation disorders & 6 & 9.8 & 30 & 2.5 & 0.001 \\
\hline \multicolumn{6}{|l|}{ Co-medications } \\
\hline Antithrombotics & 31 & 50.8 & 372 & 30.5 & 0.001 \\
\hline PPIs & 14 & 23.0 & 194 & 15.9 & 0.145 \\
\hline NSAIDs & 11 & 18.0 & 215 & 17.6 & 0.935 \\
\hline Beta-blockers & 11 & 18.0 & 117 & 9.6 & 0.032 \\
\hline Antidepressants & 10 & 16.4 & 171 & 14.0 & 0.603 \\
\hline Antiepileptic drugs & 6 & 9.8 & 69 & 5.7 & 0.175 \\
\hline Lipid-lowering drugs & 6 & 9.8 & 111 & 9.1 & 0.845 \\
\hline Calcium and/or vitamin D supplements & 6 & 9.8 & 134 & 11.0 & 0.779 \\
\hline Immunosuppressant drugs & 3 & 4.9 & 2 & 0.2 & $<0.001$ \\
\hline Corticosteroids & 2 & 3.3 & 77 & 6.3 & 0.337 \\
\hline
\end{tabular}

less than 6 months before the index date did not change the results (data not shown).

\section{Discussion}

To the best of our knowledge, this is the first investigation which estimated the risk of being exposed to oral BPs among a series of ascertained cases of ONJ. In specific, the exposure to BPs was 2.8-fold higher among patients who incurred ONJ when compared with their matched controls. In addition, although not statistically significant, a growing duration of BPs use showed an association trend toward an increased risk of ONJ occurrence.

In spite of numerous publications, very few evidence has been able to draw definite conclusions about the strength of
Table 2 Association between exposure to oral $\mathrm{BPs}$ and $\mathrm{ONJ}$

${ }^{a}$ Adjusted for diabetes, corticosteroids, PPIs

${ }^{\mathrm{b}}$ Current use: the latest date of exposure to BPs occurring within 1 year before the index date; past use: exposure to BPs interrupted more than 1 year before the index date

${ }^{\mathrm{c}}$ Cumulative use was assessed from the start date until the index date among current users

\begin{tabular}{|c|c|c|c|c|}
\hline & $\begin{array}{l}\text { Cases } \\
N=61\end{array}$ & $\begin{array}{l}\text { Controls } \\
N=1220\end{array}$ & $\begin{array}{l}\text { Crude OR } \\
(95 \% \mathrm{CI})\end{array}$ & $\begin{array}{l}\text { Adjusted OR }{ }^{\mathrm{a}} \\
(95 \% \mathrm{CI})\end{array}$ \\
\hline \multicolumn{5}{|c|}{ BPs use (any time) } \\
\hline No & $51(83.6)$ & $1102(90.3)$ & Reference & Reference \\
\hline Yes & $10(16.4)$ & $118(9.7)$ & $1.9(0.9-3.8)$ & $1.7(0.8-3.6)$ \\
\hline \multicolumn{5}{|c|}{ BPs use (recency of use) ${ }^{b}$} \\
\hline Past use & 0 & $40(3.3)$ & - & - \\
\hline Current use & $10(16.4)$ & $78(6.4)$ & $2.9(1.3-6.0)$ & $2.8(1.3-5.9)$ \\
\hline \multicolumn{5}{|c|}{ BP cumulative use (days) ${ }^{\mathrm{c}}$} \\
\hline$\leq 90$ & $4(6.6)$ & $49(4.0)$ & $1.8(0.6-5.1)$ & $1.8(0.6-5.2)$ \\
\hline $91-179$ & $2(3.3)$ & $25(2.1)$ & $1.8(0.4-8.1)$ & $1.7(0.4-7.8)$ \\
\hline $180-364$ & $2(3.3)$ & $22(1.8)$ & $2.0(0.5-9.1)$ & $1.9(0.4-8.6)$ \\
\hline$\geq 365$ & $2(3.3)$ & $22(1.8)$ & $2.0(0.5-9.1)$ & $2.0(0.4-9.8)$ \\
\hline
\end{tabular}


the association between ONJ and oral BPs, in particular when they are prescribed for preventing osteoporotic fractures. Zavras et al. [19], analyzing medical claims from approximately 20 million people in the USA, reported a fourfold increased risk of jaw surgery associated with intravenous BPs, and a nonsignificantly increased association with oral BPs. Pazianas et al. [20] used jaw surgery as a surrogate marker for ONJ and did not find any association with oral BPs. Etminan et al. [29] reported a significant relative risk of $2.87,2.43$, and 3.34 of any-site aseptic osteonecrosis for alendronate, etidronate, and risedronate, respectively. Nevertheless, none of the mentioned studies formally validated the outcome, thus making difficult to provide definite explanations to their findings.

More recently, a case-control study [21] enrolling 191 validated ONJ cases and 573 controls in 119 dental practices reported a strong association $(\mathrm{OR}=12.2$; $95 \%$ CIs, 4.335.0) between oral BPs and ONJ. However, this study included cancer patients, thus increasing the potential for residual confounding. A second limitation concerned the fact that information about medication use was obtained from telephone interviews. In these conditions, the likelihood for a recall bias could be substantial because of the high proportion of false negatives among controls, which could result in a potential overestimation of the risk [30].

In our study, we addressed these potential biases by excluding and censoring from the selected cohort patients with cancer who could be exposed to chemotherapy and/or parenteral BPs. Because of the short time window available before the "entry date" and the likelihood of underdetection of anticancer therapy issued at hospital level, we have also used very restrictive criteria for excluding the potential remaining cancer-related ONJ cases in the formal validation of medical charts. For what concerns the exposure, because it was assessed from pharmacy records, which could be regarded as a virtually complete source of information, recall bias was unlikely to occur.

Osteonecrosis of the jaw has been also associated with duration of BPs treatment, with an estimated range of 1.6 to 4.7 years, depending on BPs type [1]. However, for noncancer patients (the vast majority of whom take oral BPs), the ONJ risk seems to increase substantially after 5 years [21]. In our study, we have found a statistically significant association even after a median follow-up of 2.7 years, with cumulative BP use shorter than expected. Selective prescribing was unlikely to occur since sensitivity analyses made with propensity-matched controls showed results similar to the main analysis. Therefore, such findings might be partially explained by the inclusion into the cohort of greaterrisk patients, specifically those discharged with an osteoporotic fracture.

This association is also supported by a biological rationale. Bisphosphonates affect the physiological bone remodeling by inducing an inhibition of osteoclast recruitment, decreasing osteoclast activity, and promoting osteoclast apoptosis. This process is exerted by inhibition of the mevalonate and cholesterol biosynthetic pathways, thereby reducing the production of lipids essential for protein prenylation or posttranslational modification of the guanine triphosphates (GTP) signaling which modulates the cellular apoptosis. When GTP is uncontrolled, the osteoclast can be disrupted by an irregular cytoskeletal rearrangement, membrane ruffling, and intracellular vesicle transport [31-33]. In this way, the long-term use of nitrogen-containing BPs leads to the development of abnormal osteoclasts which degenerate into necrotic bone tissue. Moreover, BPs can exert a direct inhibitory effect on cellular proliferation and angiogenesis in the healing of alveolar bone after dental extraction [34].

Our study has several noteworthy limitations. First, although the present findings consistently support the hypothesis of a duration-response relationship, the small sample size, besides the lower than expected proportion of ONJ patients exposed to oral BPs (10/61), did not allow us to provide robust estimates about the effect of cumulative BPs use. Second, we acknowledge the possibility of residual false positives among validated cases, especially those cases with inflammatory and suppurative jaw conditions, but without any additional registered sign or symptom suggesting necrotic bone lesions or open wounds/ulcers. However, in our cohort, ONJ incidence among BPs users was 46.0 (95\% CI, 24.8.1-85.5) per 100,000 person-years, higher than $1 / 10,000-1 / 100,000$ range based on surveillance data $[7,17]$, but in agreement with two surveys conducted in Australia [14] and in the USA [18], which reported rates among patient exposed with oral BPs from 0.01 to $0.21 \%$. Furthermore, although the smaller number of cases did not permit to reach the statistical significance, the results from the sensitivity analysis conducted using only "definite" cases still indicated an increased risk among oral BP users, with a more evident cumulative effect of BPs. Thirdly, by assuming that patients who initiated the therapy had to be exposed at least 6 months to develop the ONJ, those with less than 6 months before the index date could have diluted the effect size. However, their exclusion from the analysis yielded similar results. Fourthly, the fact that ONJ was known as a BP-related disease could have introduced a detection bias among the exposed patients. Finally, some unmeasured confounders such as alcohol and smoking could have partly explained the association between the exposure and the outcome. Nevertheless, the presence of a duration-dependent association should overcome these two shortcomings.

From a clinical viewpoint, it cannot be denied that these medications have a demonstrated efficacy. In clinical trials, they have been associated with a reduction of incidence of 
fractures related to postmenopausal osteoporosis by 20 to $45 \%$ [35]. Several studies, conducted with claims databases in a "real-world setting," confirmed that greater adherence to BPs was significantly associated with a relative risk reduction of fractures after 24 months of follow-up ranging from 7 to $20 \%$ [36]. Nevertheless, although the risk of BP-related ONJ is low in non-oncological indications, it is important to be aware that it exists and to know how this clinical issue may be predicted and possibly minimized.

Acknowledgments The study was supported by Agenzia Italiana del Farmaco (AIFA grant FARM06R9YY), Rome, Italy. We would like to thank Dr. Laurent Azoulay (Department of Oncology, McGill University, Montreal, Canada) for the paper revision which he conducted according to the standard peer-review process. We would also like to thank all the other components of the BEST study group who have contributed to the scientific content or provided technical support: Emiliano Sessa (Regional Agency for Healthcare Services of Tuscany, Florence, Italy); Vincenzo Arcoraci (Department of Medicine and Pharmacology, University of Messina, Italy); Arianna Ghirardi, Lorenza Scotti, Andrea Parodi, Antonella Zambon (Unit of Biostatistics and Epidemiology, Department of Statistics, University of MilanBicocca, Milan, Italy); Carlo Piccinni, Caterina Suzzi, Aurora Puccini, Alberto Vaccheri (Department of Pharmacology, University of Bologna, Bologna, Italy); Pierangelo Geppetti (Head Unit; Center of Pharmacoutilization, Pharmacoepidemiology, Pharmacovigilance, and Pharmacoeconomics, University of Florence, Florence, Italy), Lavinia Sati (Center of Pharmacoutilization, Pharmacoepidemiology, Pharmacovigilance, and Pharmacoeconomics, University of Florence, Florence, Italy); Francesca Forlan (Department of Public Health and Environmental Medicine, University of Padua, Padua, Italy); Rosaria Gesuita (Center of Epidemiology, Biostatistics, and Medical Information Technology, Polytechnic University of Marche, Ancona, Italy); Angelo Menna (Regional Agency of Healthcare Services of Abruzzi, Chieti, Italy); Alessandra Mingarelli, Gianluca Di Tanna (Department of Public health and Infectious Disease, University "La Sapienza", Rome, Italy); and Marco Valenti (Head Unit; Department of Medicine and Public Health, University of L'Aquila, L'Aquila, Italy).

Conflicts of interest None.

\section{References}

1. Woo SB, Hellstein JW, Kalmar JR (2006) Narrative [corrected] review: bisphosphonates and osteonecrosis of the jaws. Ann Intern Med 144:753-761

2. Khosla S, Burr D, Cauley J et al (2007) Bisphosphonate-associated osteonecrosis of the jaw: report of a task force of the American Society for Bone and Mineral Research. J Bone Miner Res 22:1479_ 1491

3. Migliorati CA (2003) Bisphosphanates and oral cavity avascular bone necrosis. J Clin Oncol 21:4253-4254

4. Marx RE (2003) Pamidronate (Aredia) and zoledronate (Zometa) induced avascular necrosis of the jaws: a growing epidemic. J Oral Maxillofac Surg 61:1115-1117

5. Ruggiero SL, Dodson TB, Assael LA, Landesberg R, Marx RE, Mehrotra B (2009) American Association of Oral and Maxillofacial Surgeons position paper on bisphosphonate-related osteonecrosis of the jaws - 2009 update. J Oral Maxillofac Surg 67:2-12
6. Khan AA, Sandor GK, Dore E et al (2008) Canadian consensus practice guidelines for bisphosphonate associated osteonecrosis of the jaw. J Rheumatol 35:1391-1397

7. Assael LA (2009) Oral bisphosphonates as a cause of bisphosphonate-related osteonecrosis of the jaws: clinical findings, assessment of risks, and preventive strategies. J Oral Maxillofac Surg 67:35-43

8. Ruggiero SL, Mehrotra B, Rosenberg TJ, Engroff SL (2004) Osteonecrosis of the jaws associated with the use of bisphosphonates: a review of 63 cases. J Oral Maxillofac Surg 62:527-534

9. Marx RE, Sawatari Y, Fortin M, Broumand V (2005) Bisphosphonate-induced exposed bone (osteonecrosis/osteopetrosis) of the jaws: risk factors, recognition, prevention, and treatment. J Oral Maxillofac Surg 63:1567-1575

10. Sarasquete ME, Gonzalez M, San Miguel JF, Garcia-Sanz R (2009) Bisphosphonate-related osteonecrosis: genetic and acquired risk factors. Oral Dis 15:382-387

11. Marini F, Tonelli P, Cavalli L, Cavalli T, Masi L, Falchetti A, Brandi ML (2011) Pharmacogenetics of bisphosphonate-associated osteonecrosis of the jaw. Front Biosci (EliteEd) 3:364-370

12. Fusco V, Loidoris A, Colella G, Vescovi P, Campisi G (2010) Osteonecrosis of the jaw (ONJ) risk in breast cancer patients after zoledronic acid treatment. Breast 19:432-433

13. Vescovi P, Campisi G, Fusco V et al (2011) Surgery-triggered and non surgery-triggered Bisphosphonate-related Osteonecrosis of the Jaws (BRONJ): a retrospective analysis of 567 cases in an Italian multicenter study. Oral Oncol 47:191-194

14. Mavrokokki T, Cheng A, Stein B, Goss A (2007) Nature and frequency of bisphosphonate-associated osteonecrosis of the jaws in Australia. J Oral Maxillofac Surg 65:415-423

15. Bilezikian JP (2006) Osteonecrosis of the jaw-do bisphosphonates pose a risk? N Engl J Med 355:2278-2281

16. Edwards BJ, Gounder M, McKoy JM et al (2008) Pharmacovigilance and reporting oversight in US FDA fast-track process: bisphosphonates and osteonecrosis of the jaw. Lancet Oncol 9:1166-1172

17. Jung TI, Hoffmeister B (2007) Osteonecrosis of jaw under bisphosphonate therapy: patient profile and risk assessment. J Bone Miner Res 22:S113-S113

18. Lo JC, O'Ryan FS, Gordon NP et al (2010) Prevalence of osteonecrosis of the jaw in patients with oral bisphosphonate exposure. $\mathrm{J}$ Oral Maxillofac Surg 68:243-253

19. Zavras AI, Zhu S (2006) Bisphosphonates are associated with increased risk for jaw surgery in medical claims data: is it osteonecrosis? J Oral Maxillofac Surg 64:917-923

20. Pazianas M, Blumentals W, Miller P (2008) Lack of association between oral bisphosphonates and osteonecrosis using jaw surgery as a surrogate marker. Osteoporos Int 19:773-779

21. Barasch A, Cunha-Cruz J, Curro FA et al (2011) Risk factors for osteonecrosis of the jaws: a case-control study from the CONDOR dental PBRN. J Dent Res 90:439-444

22. Devold HM, Doung GM, Tverdal A, Furu K, Meyer HE, Falch JA, Sogaard AJ (2010) Prescription of anti-osteoporosis drugs during 2004-2007-a nationwide register study in Norway. Eur J Clin Pharmacol 66:299-306

23. Piscitelli P, Gimigliano F, Gatto S et al (2009) Hip fractures in Italy: 2000-2005 extension study. Osteoporos Int 21:1323-1330

24. Lapi F, Simonetti M, Michieli R, Pasqua A, Brandi ML, Frediani B, Cricelli C, Mazzaglia G (2011) Assessing 5-year incidence rates and determinants of osteoporotic fractures in primary care. Bone 50:85-90

25. Curtis JR, Mudano AS, Solomon DH, Xi J, Melton ME, Saag KG (2009) Identification and validation of vertebral compression fractures using administrative claims data. Med Care 47:69-72

26. Curtis JR, Taylor AJ, Matthews RS et al (2009) "Pathologic" fractures: should these be included in epidemiologic studies of osteoporotic fractures? Osteoporos Int 20:1969-1972 
27. Austin PC (2008) The performance of different propensityscore methods for estimating relative risks. J Clin Epidemiol 61:537545

28. Patrick AR, Schneeweiss S, Brookhart MA, Glynn RJ, Rothman KJ, Avorn J, Sturmer T (2011) The implications of propensity score variable selection strategies in pharmacoepidemiology: an empirical illustration. Pharmacoepidemiol Drug Saf 20:551-559

29. Etminan M, Aminzadeh K, Matthew IR, Brophy JM (2008) Use of oral bisphosphonates and the risk of aseptic osteonecrosis: a nested case-control study. J Rheumatol 35:691-695

30. Beiderbeck AB, Sturkenboom MC, Coebergh JW, Leufkens HG, Stricker BH (2004) Misclassification of exposure is high when interview data on drug use are used as a proxy measure of chronic drug use during follow-up. J Clin Epidemiol 57:973977

31. Otto S, Pautke C, Opelz C, Westphal I, Drosse I, Schwager J, Bauss F, Ehrenfeld M, Schieker M (2010) Osteonecrosis of the jaw: effect of bisphosphonate type, local concentration, and acidic milieu on the pathomechanism. J Oral Maxillofac Surg 68:2837-2845
32. Kimmel DB (2007) Mechanism of action, pharmacokinetic and pharmacodynamic profile, and clinical applications of nitrogencontaining bisphosphonates. J Dent Res 86:1022-1033

33. Hughes DE, MacDonald BR, Russell RG, Gowen M (1989) Inhibition of osteoclast-like cell formation by bisphosphonates in longterm cultures of human bone marrow. J Clin Invest 83:1930-1935

34. Diel IJ, Fogelman I, Al-Nawas B, Hoffmeister B, Migliorati C, Gligorov J, Vaananen K, Pylkkanen L, Pecherstorfer M, Aapro MS (2007) Pathophysiology, risk factors and management of bisphosphonate-associated osteonecrosis of the jaw: is there a diverse relationship of amino- and non-aminobisphosphonates? Crit Rev Oncol Hematol 64:198-207

35. Delmas PD, Siris ES (2008) NICE recommendations for the prevention of osteoporotic fractures in postmenopausal women. Bone 42:16-18

36. Siris ES, Harris ST, Rosen CJ, Barr CE, Arvesen JN, Abbott TA, Silverman S (2006) Adherence to bisphosphonate therapy and fracture rates in osteoporotic women: relationship to vertebral and nonvertebral fractures from 2 US claims databases. Mayo Clin Proc 81:1013-1022 